\title{
Next Generation Astronomical X-ray Optics: High Angular Resolution, Light Weight, and Low Production Cost
}

\author{
W.W. Zhang ${ }^{a}$, M.P. Biskach ${ }^{b}$, P.N. Blake, K.W. Chan ${ }^{\mathrm{c}}$, J.A. Gaskin ${ }^{\mathrm{d}}$, M.L. Hong ${ }^{b}$, W.D Jones ${ }^{\mathrm{d}}$, \\ L.D. Kolos ${ }^{\mathrm{a}}$, J.R. Mazzarella ${ }^{\mathrm{b}}$, R.S. McClelland ${ }^{\mathrm{b}}$, S.L. O'Dell ${ }^{\mathrm{d}}$, T.T. Saha ${ }^{\mathrm{a}}$, and M.V. Sharpe ${ }^{\mathrm{b}}$ \\ a NASA Goddard Space Flight Center, Greenbelt, MD 20771 \\ ${ }^{b}$ Stinger Ghaffarian Technologies, Inc., Greenbelt, MD 20770 \\ ${ }^{c}$ University of Maryland-Baltimore County, Baltimore, MD 21250 \\ ${ }^{d}$ NASA Marshall Space Flight Center, Huntsville, AL 35812
}

\begin{abstract}
X-ray astronomy depends on the availability of telescopes with high resolution and large photon collecting areas. Since $x$-ray observation can only be carried out above the atmosphere, these telescopes must be necessarily lightweight. Compounding the lightweight requirement is that an $\mathrm{x}$-ray telescope consists of many nested concentric shells, which further require that x-ray mirrors must also be geometrically thin to achieve high packing efficiency. This double lightweight and geometrically thin requirement poses significant technical challenges in fabricating the mirrors and in integrating them into mirror assemblies. This paper reports on the approach, strategy and status of our x-ray optics development program whose objective is to meet these technical challenges at modest cost to enable future $\mathrm{x}$-ray missions, including small Explorer missions in the near term, probe class missions in the medium term, and large flagship missions in the long term.
\end{abstract}

Keywords: X-ray optics, lightweight optics, glass slumping, mirror alignment, mirror bonding

\section{Technological Challenges}

An ideal x-ray telescope [1, 2], or telescope of any kind, would have an exquisite angular resolution to facilitate the imaging of the finest details of astronomical objects, and would have a large photon collection area to allow the observation of some of the faintest objects with a relatively short exposure time. There are three obstacles to the realization of an ideal x-ray telescope. First, since astronomical x-ray observation can only be carried out in space above Earth's atmosphere, the launch capacity and cost of existing rockets pose severe limitations on both mass and volume of such an observatory. One has to work within the general perimeter set by available launch vehicles that are not specifically designed and built for purposes launching $\mathrm{x}$ ray telescopes. In general, launch cost is proportional to the mass and volume of the payload. In this regard, an x-ray telescope must be as light and as small as possible, given a specific set of observational performance requirements in terms of angular resolution, effective area, and energy bandwidth. Second, the grazing incidence nature of $\mathrm{x}$-ray optics requires the nesting of a large number of concentric shells to achieve a desired photon collection area. Each of these concentric shells must have a very low areal density to minimize the mass of the telescope and must be as thin as possible to achieve efficient packing. This is in direct contrast with space telescopes in the visible band where mirrors need only to have a low areal density, but do not 
have to be geometrically thin. Third, the grazing incidence nature further dictates that even a modest photon collection area requires a large physical mirror area. This again is in direct contrast with an optical telescope where photon collection area is nearly synonymous to physical mirror area. In a nutshell, the challenge of making a future $\mathrm{x}$-ray telescope is two-fold: (1) fabrication of large quantities of lightweight and thin mirrors, and (2) assembly of these mirrors into a telescope.

\section{DeVELopMent STRategy}

A future telescope could be a large flagship along the line of Chandra [3], XMM-Newton [4], and IXO [5] that are designed to address broad areas of astrophysics and cosmology. Or it could also be much smaller ones dedicated to the study of a particular area of astrophysics, like a small explorer mission. We have adopted the segmented approach, shown in Figure 1, in which a telescope mirror assembly is divided into a number of small modules each of which can be built and tested separately and then integrated to make up the whole telescope. The advantages of the segmented approach are manifold. It diminishes the difference between building a large mirror assembly and a small mirror assembly. A large mirror assembly mainly means the construction and integration of more modules. It does not entail new and custom-designed and -built equipment, which can be extremely time-consuming and expensive. The segmented approach easily lends itself to mass production. A small mirror assembly, up to a diameter of $0.5 \mathrm{~m}$, requires $\sim 10$ identical modules. A large mirror assembly may mean dozens of modules of two or three distinct types. This makes the management of spares and production significantly easier and therefore lowers the overall cost and schedule of manufacturing a mirror assembly.

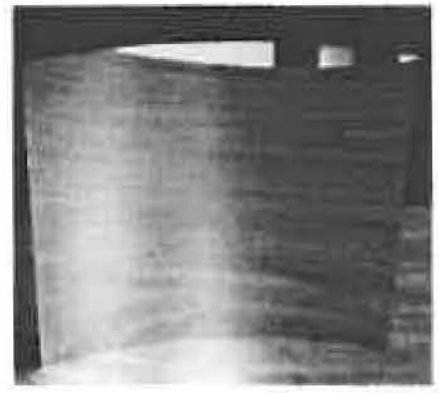

Mirror Segment

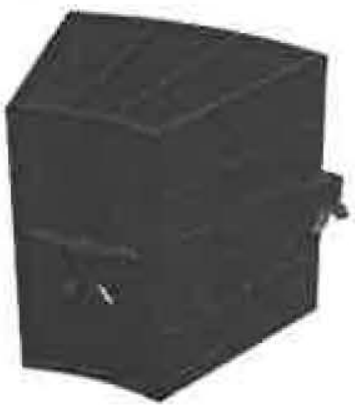

Mirror Module

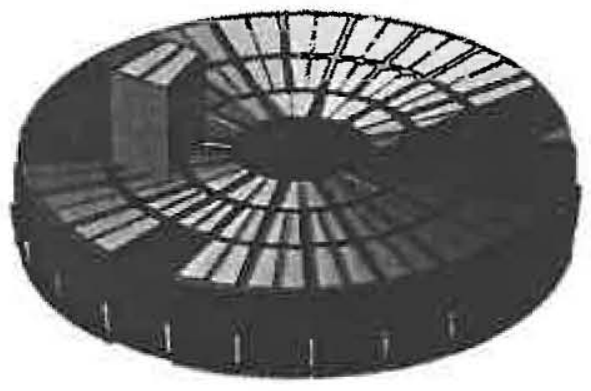

Telescope

Figure 1. Hierarchical structure of a segmented design: mirror segment, mirror module, and telescope mirror assembly. Nominally, a mirror segment is $200 \mathrm{~mm} \times 200 \mathrm{~mm}$; a mirror module contains $\approx 100 \mathrm{co}-$ aligned mirror pairs. (primary and secondary); and a telescope mirror assembly comprises of order 100 aligned and integrated modules.

Our technology development strategy is to maximize the use of commercially available material and equipment. This not only minimizes the cost and schedule of making a telescope, it also minimizes the cost of technology development. It makes possible a vibrant technology development program under a severely constraining budgetary environment. 
Suzaku[6] and NuSTAR[7] represent the state of the art of lightweight x-ray telescope making. They have angular resolutions of approximately 120 and 60 arc-seconds, respectively. The best angular resolution among $\mathrm{x}$-ray telescopes is Chandra's 0.5 arc-seconds, which was realized by polishing thick Zerodur mirror shells at a very high cost. As we embark on the task of developing a technology of building lightweight telescopes with high angular resolutions, it is wise to recognize that it probably is unrealistic to achieve more than an order or magnitude improvement in angular resolution in a single step. As such, we have adopted a two-phase approach. In the first phase, to be realized in the next couple of years, we want to make ready a technology that can make lightweight telescopes with an angular resolution of $\sim 5$ arc-seconds. It will enable a number of Explorer missions as well as missions that are descendants of the IXO mission concepts.

In the second phase, or a longer tem, i.e., 3 to 10 years, the objective of our development is to achieve sub-arc-second angular resolution at mass per unit area of Suzaku and cost affordable by an Explorer mission. By design our technical approaches meet both lightweight and cost requirements. They are capable of making telescopes at the same mass per unit effective area as that of Suzaku for a soft band $(0.1-10 \mathrm{keV})$ telescope and that of NuSTAR for a hard band one $(\sim 5-100 \mathrm{keV})$. We seek to develop and refine each technique to achieve angular resolution requirements verified by repeated $\mathrm{x}$-ray performance tests. Then we engineer the techniques to meet spaceflight environmental requirements, such as vibration, acoustic, and thermal vacuum requirements. Last but not least, we seek to streamline each step of the process to achieve maximum simplicity and efficiency and thereby minimize both cost and schedule for making mirror modules.

\section{TeChNical Approaches and Status}

The entire process of building a mirror assembly starts with the making of mirror substrates, which are then coated with a thin film $(\sim 20 \mathrm{~nm})$ of a noble metal such as iridium to maximize reflectivity. We have developed and perfected a glass slumping process $[8,9,10,11,12,13,14$, $15,16,17,18]$, as shown in Figure 2. It copies the figure of conventionally fabricated mandrels to commercially procured (Schott D263) thin $(\sim 0.4 \mathrm{~mm})$ float glass sheets while preserving their excellent micro-roughness ( $\sim 4 \AA \mathrm{rms}$ measured over a $300 \mu \mathrm{m}$ span). As shown in Figure 2 (right panel), it has been able to make mirror substrates consistently at $\sim 6.5$ arc-seconds (HPD). Each entry in the histogram represents a substrate pair which has been precisely measured with optical metrology $[19,20,21,22,23,24]$ and whose $x$-ray imaging performance calculated with standard performance prediction techniques. The glass slumping process is capable of making substrates the meet the requirements of a sub-10 arc-second mirror assembly. It has been used to making the more than 10,000 substrates for the NuSTAR mission [7]. 

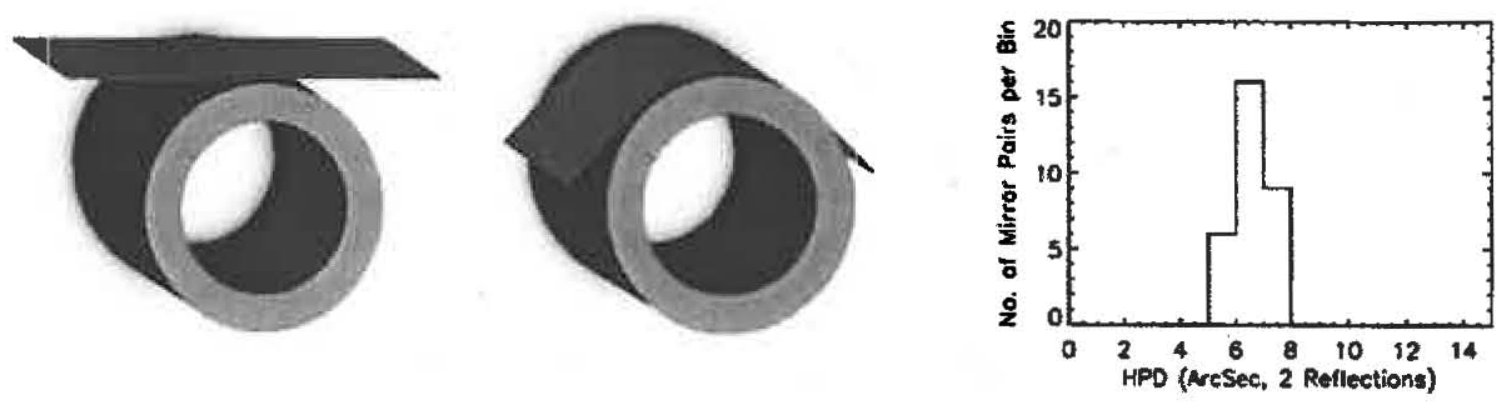

Figure 2. Left and middle: In the glass slumping process, a thin float-glass sheet slumps under its own weight as the temperature ramps gradually to $\sim 600^{\circ} \mathrm{C}$, replicating the mandrel's precise figure. Right: Histogram of the figure quality of 32 pairs of consecutively produced mirrors gives a mean imaging quality is 6.5-arcsecond HPD (two reflections), satisfying the allocation for making a 10-arcsecond telescope.
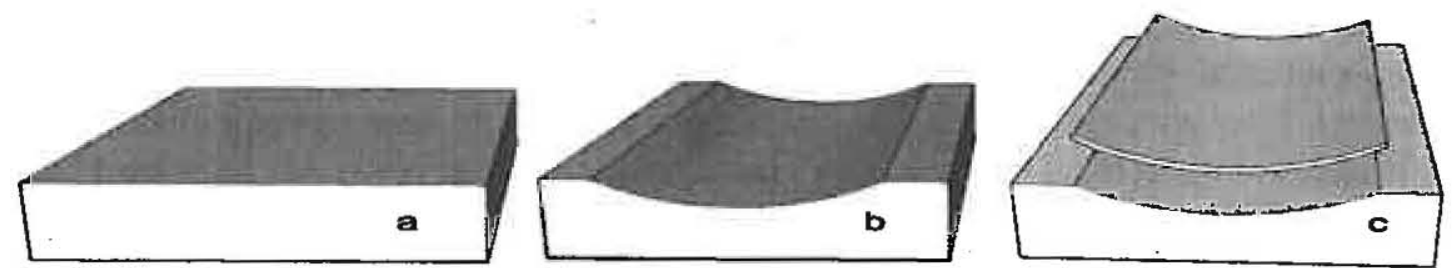

Figure 3. Illustration of the three steps for making a high-resolution Wolter-I mirror substrate: (1) A block of monocrystalline silicon, properly cut and etched to remove all subsurface damage caused by the cutting; (2) a conical shape is directly cut (using a wire-EDM process or a diamond-studded wire-saw) and precision figured and polished after removing subsurface damage with a chemical etch; and (3) the mirror is sliced off the silicon block and the back (convex) surface is etched to remove subsurface damage. The resulting mirror substrate is expected to have the same figure as before slicing because the mirror substrate is still a single crystal free of any stress.

In the past year we have started the development of a new mirror substrate fabrication process, shown in Figure 3. It is intended to take advantage of two developments since the fabrication of the Chandra mirrors: (1) the availability of large blocks of single crystal silicon at affordable prices, and (2) commercial standardization of mirror polishing and finishing techniques that are capable of achieving excellent figure quickly and inexpensively. In comparison with the Schott D263 glass or other materials, single crystal silicon has several distinct advantages. Its thermal conductivity is 100 times higher and its coefficient of thermal expansion 2 times lower, making it a much easier to provide a thermal environment for its operation. Its elastic modulus is twice that of the D263 glass, making it much less susceptible to distortion caused by gravity and handling. Its density is approximately $10 \%$ lower than D263 glass. The most important property, however, is that, being a nearly perfect single crystal where each atom is in its proper location, single crystal silicon is free of internal stress. This lack of internal stress makes is possible for us to figure a mirror segment and then lightweight it to the desired thickness, as shown in Figure 3, provided that any surface and subsurface damage caused by the light-weighting process can be properly removed by acid etch and/or healed by annealing. 


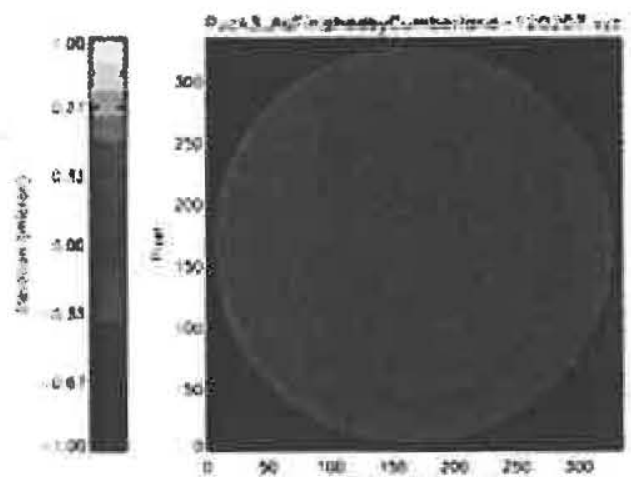

Figure beforè slicing
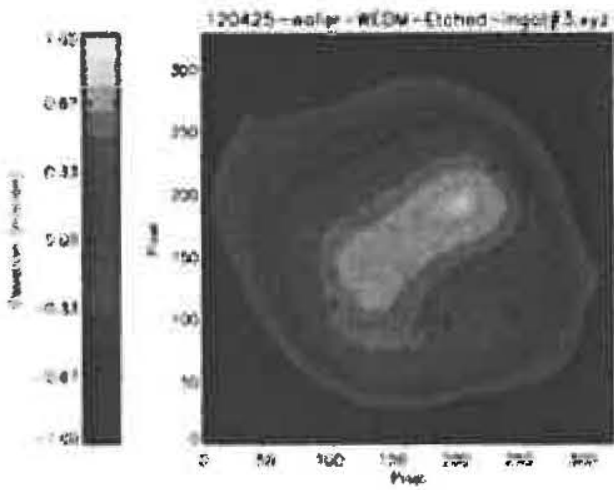

After acid etch of back

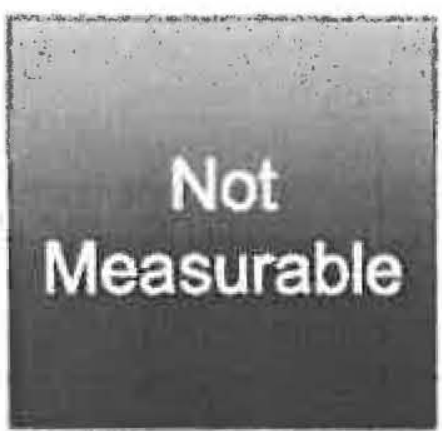

After slicing
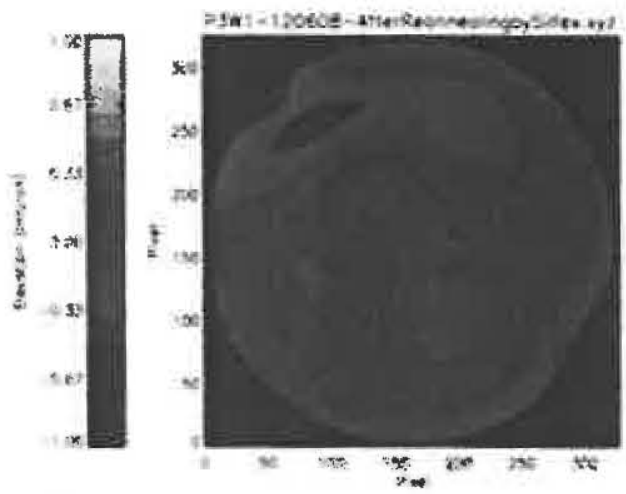

After re-annealing

Figure 4. Figure degradation and improvement of a flat silicon mirror. Upper-left: an 8-inch in diameter and 2-inch thick mirror; Upper-right: the "figure" of a 4-mm thick wafer sliced off the 8-inch mirror, the distortion caused by the wire-EDM slicing process being so bad that the figure cannot be measured; Lowerleft: the same wafer after a acid etch process which removed $\sim 0.1 \mathrm{~mm}$ from the back surface; Lower-right: the same wafer after an annealing process which further healed subsurface damage.

We are in the process of proving the concept outlined in Figure 3. To expedite and minimize the cost of the process, instead of using a parabolic mirror we use flat mirrors. Figure 4 shows an initial result. We started with a single crystal silicon disk, $210 \mathrm{~mm}$ in diameter and $55 \mathrm{~mm}$ thick. After it is properly annealed, it is polished flat by a vendor using a traditional planetary polisher. Its figure measurement on a Zygo interferometer is shown in the upper-left panel of Figure 4. Then a 5-mm wafer was sliced off using a wire electric-discharge machine. Because of subsurface damage, the figure of the surface is completely lost. It is so out of flatness that it cannot be measured at all on the interferometer, as shown in the upper-right panel. Then the wafer, with its polished side protected by a thick layer of wax, was etched in an acid to remove the subsurface damage and it recovered its figure somewhat and can be measured, as shown in the lower-left panel of Figure 4. As a last step, the wafer was annealed again at $\sim 1,250^{\circ} \mathrm{C}$. The figure becomes significantly better, as shown in the lower-right panel. The result shown in Figure 4 demonstrates that at least qualitatively the process outlined in Figure 3 could work. But the loss of figure quality is too much to be acceptable. At the present time, we believe that some of the final loss of figure has been due to unexpected subsurface damage on the polished surface 
itself. In future trials we will ensure by fine polishing that the mirror surface is totally free of surface and subsurface damage. Our plan is that, as soon as we have demonstrated the concept works with flat mirrors, we will make parabolic and hyperbolic mirrors.

Once a mirror substrate is made, it needs to be coated to maximize its x-ray reflectivity [25]. While exactly what material to coat at what thickness is a subject of intense study to achieve truly maximum reflectivity, in general $20 \mathrm{~nm}$ of a noble metal is sufficient. For the typical energy band of interest, iridium is the best material to use. The coating must meet three requirements: preserving the micro-roughness of the substrate, achieving near bulk density, and preserving the figure of the substrate. In general the first two requirements are easy to meet. It is the third one that is difficult to achieve for a very thin mirror substrate. In general thin film is highly stressed. The sign (whether compressive or tensile) and the magnitude of the stress are material- and process-dependent. We have been investigating both the magnetron sputter process and the atomic layer deposition (ALD) process. They each have its advantages and disadvantages. Recently we have been more attracted to ALD for its capability of coating both the front (concave) and back (convex) sides of the mirror simultaneously and with the same thickness. This property has the potential of achieving balance of the stresses from the two sides, resulting in no net distortion to the mirror substrate. Our investigation of the coating process continues into the next year and we expect to choose one of the two processes for refinement to meet all of the three requirements.

(b)

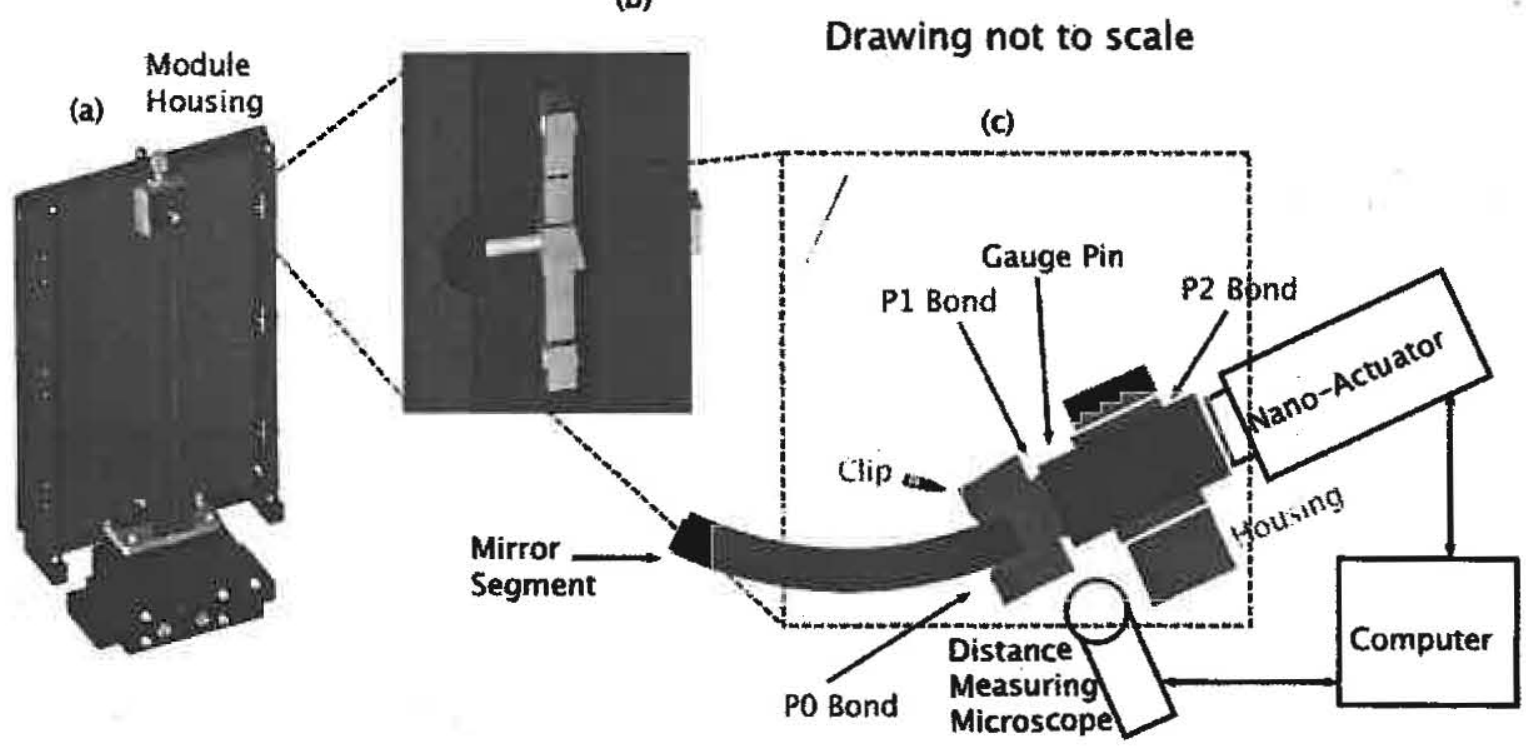

Figure 5. A schematic illustration of the way each mirror segment is bonded to the module housing. (a) Each mirror is bonded at 6 locations to the housing to give it the ability to withstand launch loads; (b) a zoomed-in view of a bond; (c) further zoomed-in details of a bond, consisting of three adhesive bonds: P0, P1, and P2. The nano-actuator, distance-measuring microscope, and computer are equipment used to perform the bonding and are not part of the finished mirror module.

Once a mirror substrate is measured and coated to become a mirror segment, it is measured again to ensure that it, while free-standing and undistorted, meets all requirements, including figure, micro-roughness, and focal length. It is then aligned using an optical Hartmann system to the 
mirror module housing and to other mirror segments. Once an optimal alignment is achieved, judging by both focus quality and light intensity, it is bonded to the housing $[26,27,28,29]$. Each mirror segment is bonded to the housing at six locations, which, according to detailed finite element analysis [30], will enable the mirror segment to withstand launch loads. The details of the bonding process are illustrated in Figure 5. The first step of the bonding process is the attachment of six clips with an adhesive to the mirror edges to distribute the load. (The actual attachment of these clips happens before the mirror segment is delivered to the alignment and assembly process. It is accomplished as part of the mirror segment preparation process.) The attachment of the mirror to the housing is accomplished by precision gauge pins and precisely lapped holes in the housing walls. The pin and the hole are closely matched such that the clearance between them is minimal. Once the mirror achieves alignment, a pin with a dab of epoxy on its tip is inserted into the hole under the monitoring and control of a distancemeasuring microscope and a nano-acturator. Once the tip of the pin is determined to have touched the clip surface, a small amount of adhesive is wicked into the clearance between the pin and the hole. The mirror segment is firmly attached to the housing when both the P1 and P2 joints have fully cured.
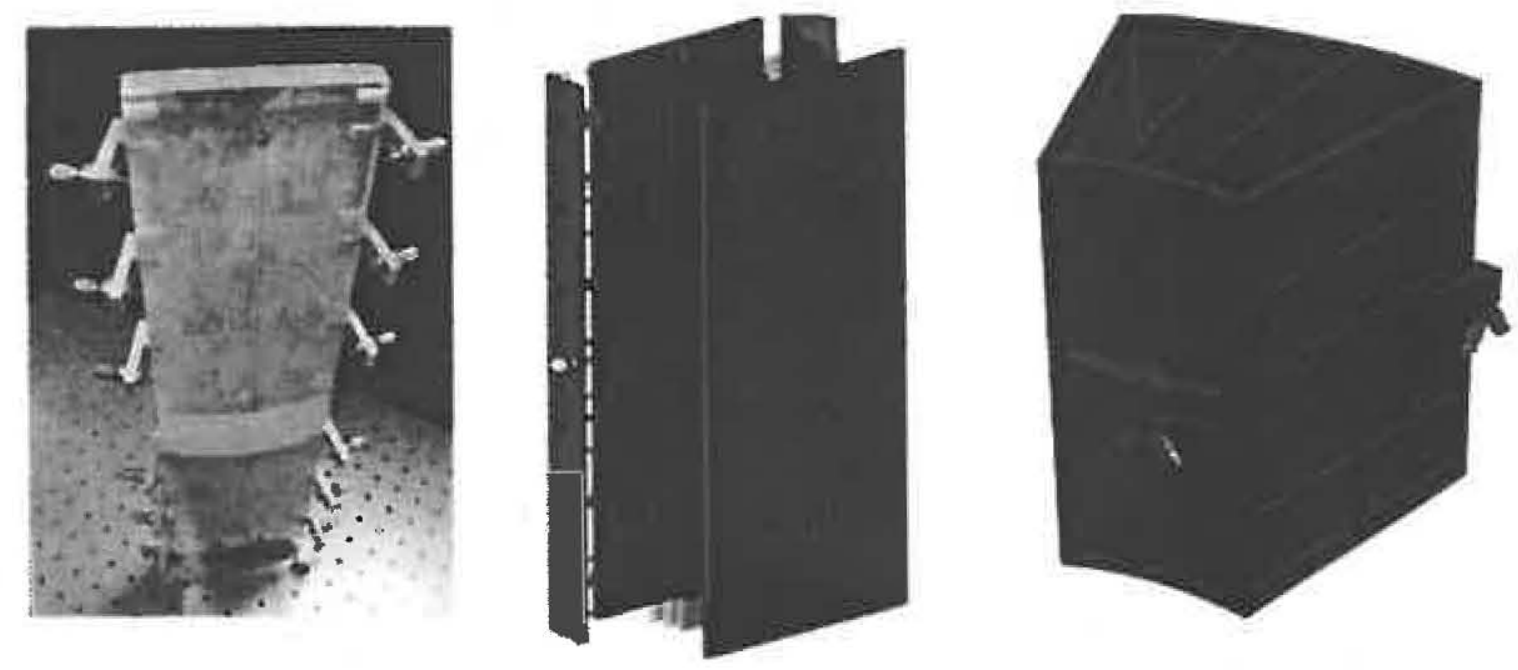

Figure 5. Three types of modules depicting the progression of our technology development. Left: a mirror housing simulator to which single pairs of mirrors are aligned and bonded; Middle: an expanded view of a mini-module that contains three co-aligned and bonded mirror pairs; Right: a high-fidelity module that has tens of to hundreds of mirror segments aligned and bonded.

This alignment and bonding process has been developed and tested in the last year. It has been used to bonding numerous mirror segments each by themselves first and then one pair at a time. Figure 5 shows the progression of our alignment and bonding work. The mirror segment bonding technique has been validated using the relatively crude and simple fixture shown in the left panel. In recent months, it has been used to co-align and bond multiple mirror segments into housings as shown in the middle panel of Figure 5. One of finished technology development modules, with 3 pairs of mirror segments co-aligned and bonded, is shown in Figure 6a while it is being tested in an $\mathrm{x}$-ray chamber. Figure $6 \mathrm{~b}-\mathrm{d}$ show the result of a full-illumination $\mathrm{x}$-ray test. 

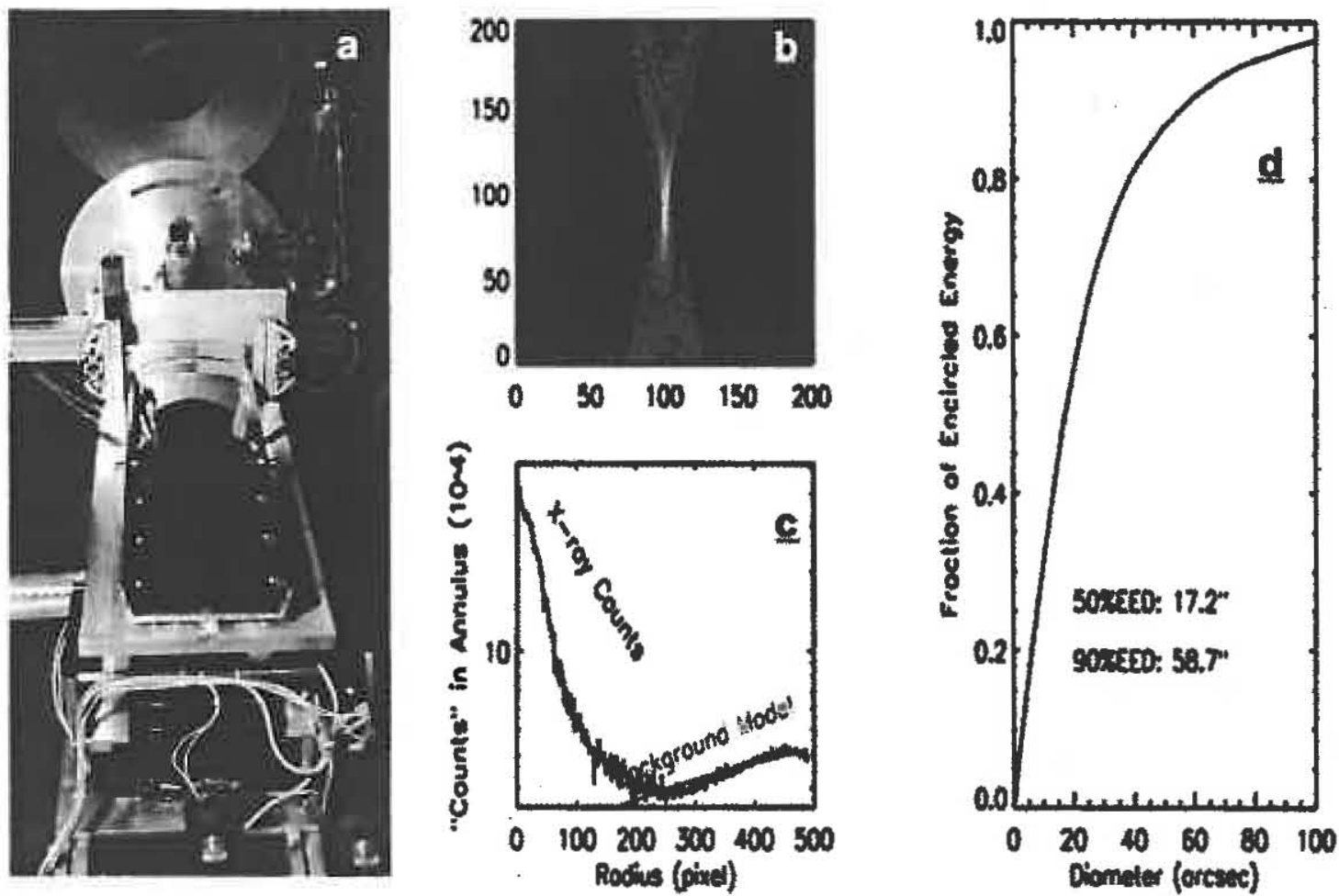

Figure 6. A summary of a recent $x$-ray test of one of two technology development modules, each of which contains three pairs of parabolic-hyperbolic mirror segments co-aligned and bonded to a housing structure. (a) A photo of the TDM set in a vacuum chamber a the end of a 600-m beam line at NASA Goddard Space Flight Center; (b) a typical x-ray image obtained with $4.5 \mathrm{keV}$ x-rays; (c) the same $\mathrm{x}$-ray image decomposed into number of counts in an annulus as a function of distance from the center of the image with the red curve showing background estimates; and (d) Fraction of encircled energy as a function of the image diameter, showing the 3-pair module has a half-power diameter of 17.2 arc-seconds.

\section{Prospects}

We have made significant progress toward the goal of building high resolution, lightweight, and low cost x-ray telescopes, but much remains to be done. First of all, as shown in Figure 6, the xray imaging quality of $\sim 17$ arc-seconds HPD (half-power diameter) is still dominated by figure distortions that arise during the mirror segment bonding process. Of several potential causes of the distortions, two stand out: thermal distortion that has been frozen in during the epoxy bonding process and randomness of epoxy cure process. Our effort in the next year will be focused on these causes. After we have adequately addressed them we expect to be able to realize the full potential of the mirror substrates, constructing technology development modules capable of producing images better than 10 arc-seconds HPD. An integral part of this process is to finalize the coating process [25] to minimize mirror figure degradation resulting from coating stress. Once the full potential of the slumped glass mirror substrates ( 6.5 arcseconds) has been realized, we expect that the mirror substrates fabricated from single crystal silicon will become available to a quality close to or even better than one arc-second. The better silicon mirror substrates will in turn stimulate the development of better alignment and bonding techniques. In the long run we expect that successively better substrates and bonding techniques will lead to the construction of mirror modules of angular resolution better than one arc-second.

Meanwhile we have begun to conduct rigorous dynamic analysis and environmental tests of the technology development modules, including vibration, acoustic, thermal vacuum, and shock tests 
$[30,31]$, in addition to rigorous $x$-ray performance tests that will be conducted at Marshall Space Flight Center. Our objective is to establish and demonstrate a complete process, both analytically and empirically, of constructing mirror modules that have better than 10 arc-second resolution in the near term (one to two years) and sub-arc-second resolution in the long term (three to ten years).

\section{ACKNOWLEDGEMENTS}

The work described in this paper represents the effort of many people over several years. The authors wish to acknowledge the support of the many scientists, engineers, and technicians both at NASA Goddard Space Flight Center (GSFC) and at NASA Marshall Space Flight Center (MSFC). The work has been financially supported by NASA through the Physics of the Cosmos Program Office at GSFC and through an Astronomy \& Physics Research and Analysis Program (APRA) grant under the Research Opportunities in Space and Earth Sciences (ROSES) program.

\section{REFERENCES}

1. Zhang, W., et al., "Generation-X: a large aperture, high angular resolution $x$-ray observatory for the 2010's", ASPC 234, 657-662 (2001)

2. Windhorst, R.A., et al., "Generation-X: an $x$-ray observatory designed to observe first light objects", NewAR 50, 121-126 (2006)

3. Gordon, T.E., \& Catching, B. F., "Status of the Advanced X-Ray Astrophysics Facility (AXAF) optics production program", SPIE 2263, 233-242 (1994)

4. Gondoin, P., et al. "X-ray multi-mirror (XMM) telescope", SPIE 2279, 86-100 (1994)

5. Bookbinder, J. et al., "The International X-ray Observatory," AIPC, Vol. 1248, pp. 561$566(2010)$

6. Serlemitsos, P. J., et al. "The $x$-ray telescope onboard Suzaku", PASJ 59, 9-21 (2007)

7. Craig, W.W. et al., "Fabrication of the NuSTAR flight optics, " SPIE 8147 (2011)

8. Zhang, W.W. et al., "Lightweight and high angular resolution $x$-ray optics for astronomical missions, " SPIE 8147 (2011)

9. Zhang, W.W., et al., "Mirror technology development for the International X-ray Observatory mission (LXO), " SPIE 7732 (2010)

10. Zhang, W.W. et al., "Mirror technology development for the International X-Ray Observatory (LXO) mission, " SPIE, 7360 (2009)

11. Zhang, W.W. et al., "Constellation-X mirror technology development," SPIE 7011 (2008)

12. Zhang, W.W. et al., "Development of lightweight $x$-ray mirrors for the Constellation- $X$ mission," SPIE 6688 (2007)

13. Zhang, W.W. et al., "Constellation-X mirror technology development," SPIE 6688 (2007)

14. Zhang, W.W. et al., "Development of lightweight $x$-ray mirrors for the Constellation- $X$ mission, "SPIE 6266 (2006)

15. Zhang, W.W. et al., "Development of lightweight $x$-ray mirrors for the Constellation-X mission, " SPIE 5900 (2005)

16. Zhang, W.W. et al., "Development of lightweight $x$-ray mirrors for the Constellation- $X$ mission," SPIE 5488 (2004)

17. Zhang, W.W. et al., "Development of $x$-ray reflectors for the Constellation- $X$ observatory," SPIE 5168 (2004) 
18. Zhang, W.W., et al. "Development of mirror segments for the Constellation- $X$ observatory", SPIE 4851 (2003)

19. Lehan, J.P. et al., "Design and fabrication of refractive nulls for testing the segmented mirrors of the Constellation-X spectroscopy $x$-ray telescope (SXT), " SPIE 5900 (2005)

20. Lehan, J.P. et al., "Toward a complete metrologic solution for the mirrors for the Constellation- $X$ Spectroscopy $x$-ray telescope, "SPIE 6688 (2007)

21. Lehan, J.P., et al., "Progress toward a complete metrology set for the International X-ray Observatory (LXO) soft x-ray mirrors", SPIE 7437 (2009)

22. Saha, T.T., et al., "Grazing incidence wavefront sensing and verification of $x$-ray optics performance, " SPIE 8147 (2011)

23. Chan, K.-W. et al., "Metrology of LXO mirror segments, " SPIE 8147 (2011)

24. Lehan, J.P. et al., "Some considerations for precision metrology of thin $x$-ray mirrors," SPIE 7018 (2008)

25. Chan K.-W. et al., "Reflective coating for lightweight x-ray optics," SPIE 8443 (2012)

26. Biskach, M.P. et al., "Precise alignment and permanent mounting of thin and lightweight $X$-ray segments, " SPIE 8443 (2012)

27. Chan, K.-W. et al., "Mounting and alignment of LXO mirror segments, " SPIE 7732 (2010)

28. Chan, K.-W. et al., "Opto-mechanics of the Constellation-X SXT mirrors: challenges in mounting and assembling the mirror segments, "SPIE, Vol. 7011 (2008)

29. Chan, K.-W. et al., "Mechanical and thermal analysis of the spectroscopy $x$-ray telescopes for the Constellation-X mission, "SPIE 6688 (2007)

30. McClelland, R.S. et al., "Design and analysis of mirror modules for $L X O$ and beyond," SPIE 8147 (2011)

31. McClelland, R.S. et al., "Design and Analysis of Modules for Segmented X-Ray Optics," SPIE 8443 (2012) 